\title{
Adrenal insufficiency from bilateral adrenal haemorrhage in a postoperative patient on warfarin
}

\author{
Emily T. Mudenha, M. Rathi \\ King's Mill Hospital, Sutton-in-Ashfield
}

\section{Introduction}

We present a case which highlights the non-specific nature of the presenting symptoms of adrenal failure due to bilateral adrenal haemorrhage.

\section{Case report}

A 75year old gentleman had an uncomplicated right total hip replacement for osteoarthritis and his warfarin for atrial fibrillation was restarted on the third post-operative day before discharge. A week later, he was admitted with watery diarrhoea and vomiting. On examination, he was clinically dehydrated, blood pressure $96 / 64 \mathrm{mmHg}$ and mild peri-umbilical tenderness. Laboratory tests revealed; sodium 130mmol/L (134-145), potassium $5.6 \mathrm{mmol} / \mathrm{L}(3.5-5.3)$, urea $14.8 \mathrm{mmol} / \mathrm{L}$ (baseline 6) and creatinine 171umol/L (baseline 80). INR was 4.6.

He was adequately resuscitated, managed as likely gastroenteritis and discharged when he stabilised. He was then readmitted another two weeks later, dehydrated and hypotensive with potassium of $7.6 \mathrm{mmol} / \mathrm{l}$ and sodium $132 \mathrm{mmol} / \mathrm{l}$. His potassium was adequately treated but as the gentleman had persisting symptomatic hypotension he then had a short synacthen test which revealed baseline serum cortisol $62 \mathrm{nmol} / \mathrm{L}$ and post synacthen cortisol $63 \mathrm{nmol} / \mathrm{L}$, confirming a diagnosis of adrenal insufficiency. His antibodies were negative and an adrenal MRI scan confirmed bilateral adrenal haemorrhage.

He was then started on appropriate steroid replacement and fludrocortisone. His warfarin however was never discontinued.

\section{Discussion}

Adrenal failure due to bilateral adrenal haemorrhage is very rare and its pathophysiology is poorly understood. It may be of a multifactorial aetiology. Several pathophysiological changes during the intraoperative and postoperative period make the adrenal gland susceptible to adrenal haemorrhage. These may include intraoperative hypotension, vascular engorgement and stasis which cause an increase in adrenal venous pressure. The diagnosis of adrenal insufficiency secondary to adrenal haemorrhage is often delayed because of the nonspecific nature of the clinical presentation and low index of clinical suspicion. There is a need for clinicians to be aware of this potentially life-threatening but readily treatable condition.

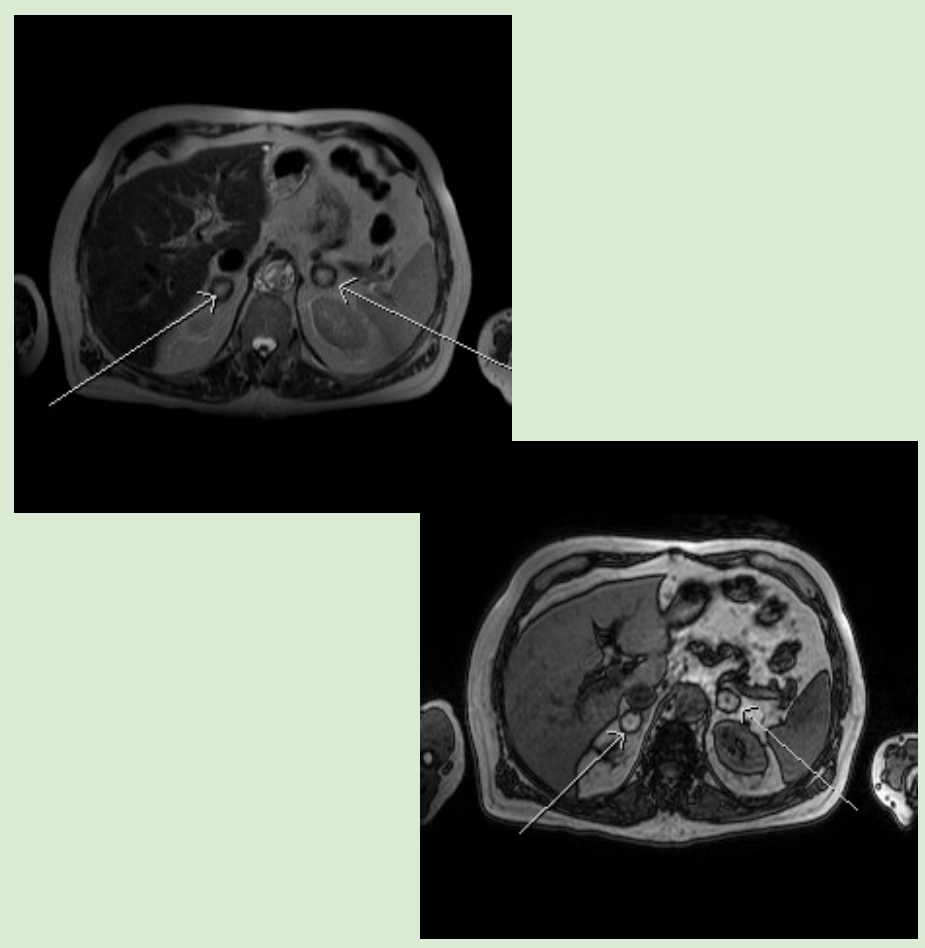

